PROCEEDINGS OF THE

AMERICAN MATHEMATICAL SOCIETY

Volume 129, Number 8, Pages 2227-2234

S 0002-9939(01)05905-6

Article electronically published on February 2, 2001

\title{
ON BIRATIONAL MORPHISMS BETWEEN PENCILS OF DEL PEZZO SURFACES
}

\author{
VITALY VOLOGODSKY
}

(Communicated by Ron Donagi)

\begin{abstract}
Let $X / S$ and $X^{\prime} / S^{\prime}$ be two Del Pezzo fibrations of degrees $d, d^{\prime}$ respectively. Assume that $X$ and $X^{\prime}$ differ by a flop. Then we prove that $d=d^{\prime}$ and give a short list of values of other basic numerical invariants of $X$ and $X^{\prime}$.
\end{abstract}

\section{IntRoduction}

After successful completion of the Minimal Model Program in dimension 3 an attempt to apply this theory to the factorization of birational morphisms of rational algebraic varieties was made in a series of works [Sar, Re, Cor. The objects of a new category $\mathcal{S}(3)$ introduced by Sarkisov are three-dimensional $\mathbb{Q}$-Fano fiber spaces $\alpha: X \rightarrow S$, and arrows are birational maps between them:

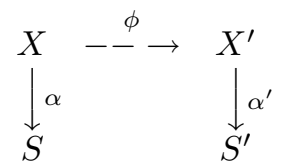

Four types of elementary arrows, or links, in $\mathcal{S}(3)$ were defined. According to Cor every arrow in $\mathcal{S}(3)$ is a composition of elementary links. Little is known about the precise structure of elementary links and their classification is of great interest.

This work is an attempt to investigate elementary links of type (IV) which, by definition, are the commutative diagrams of the following type:

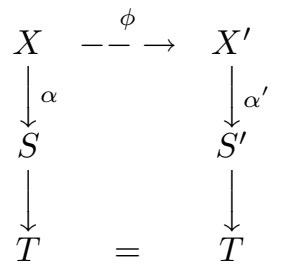

Here $\phi$ is a sequence of log-flips and $\rho(S / T)=\rho\left(S^{\prime} / T\right)=1$.

We will work with the case of two Del Pezzo fibrations. The simplest possible situation is when $X$ and $X^{\prime}$ are smooth and the map $\phi$ is a flop. Then our diagram

Received by the editors May 27, 1998 and, in revised form, December 23, 1999.

2000 Mathematics Subject Classification. Primary 14E05; Secondary 14E30, 14 E35.

(C)2001 American Mathematical Society 
looks like this:

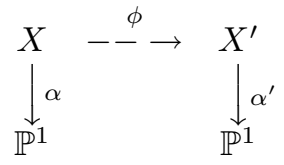

and $\rho(X)=\rho\left(X^{\prime}\right)=2$.

In this case varieties $X$ and $X^{\prime}$ are similar to Fano threefolds. The anti-canonical class $\left(-K_{X}\right)$, resp. $\left(-K_{X^{\prime}}\right)$, is not ample but it is nef and big. Using calculation in Chow rings we find a list of values of some basic numerical invariants.

Theorem 0.1. If $X$ and $X^{\prime}$ are two smooth Del Pezzo fibrations, $d$ is the degree of the fibration $X \rightarrow \mathbb{P}^{1}$, and the birational map $\phi: X--\rightarrow X^{\prime}$ is a flop, then the list of the possible values for $K_{X}^{3}$ and $d$ is limited to the following:

1) $K_{X}^{3}=-54, d=9$;

2) $K_{X}^{3}=-32, d=8$;

3) $K_{X}^{3}=-2 n, d=n$, for $1 \leq n \leq 9, n \neq 7$;

4) $K_{X}^{3}=-4, d=4$;

5) $K_{X}^{3}=-2, d=3$.

The most important corollary is that the degrees of the two Del Pezzo fibrations coincide. In the last section we give examples for some cases from the list.

\section{Preliminary Results}

Because of the smoothness of $X$ and $X^{\prime}$ their groups of Weil and Cartier divisors coincide. As $\phi$ is an isomorphism in codimension one, we have a one-to-one correspondence between irreducible divisors on $X$ and $X^{\prime}$. Therefore, $\operatorname{Pic} X \simeq \operatorname{Pic} X^{\prime}$.

Proposition 1.1. $\phi$ is not an isomorphism.

Proof. Assume $X \simeq X^{\prime}$. Now $X$ is smooth and by Poincaré duality the Mori cone of curves will be two-dimensional. Two extremal rays correspond to morphisms $\alpha$ and $\alpha^{\prime}$ and they have negative intersection with $K_{X}$. Therefore, every effective 1-cycle has negative intersection with $K_{X}$; that is, $X$ is a Fano variety. If $F$ is a fiber of $\alpha$ and $F^{\prime}$ is a fiber of $\alpha^{\prime}$, then the divisors $F, F^{\prime}$ are linearly independent in Pic $X$. We have $F^{2} \equiv 0,{F^{\prime}}^{2} \equiv 0$ and $K_{X} F F^{\prime} \neq 0$. This means that $K_{X}$ cannot be a linear combination of $F$ and $F^{\prime}$, and $\rho(X) \neq 2$. Contradiction.

Remark 1.2. Proposition 1.1 also can be derived from the classification of threedimensional Fano varieties [MM]. There are six smooth Fano varieties $X$ with $\rho(X)=2$ which have two structures of $\mathbb{Q}$-Fano fibrations. But at least one of these fibrations is a conic bundle.

Note that the anti-canonical class $\left(-K_{X}\right)$ is nef, and so is $\left(-K_{X^{\prime}}\right)$ by symmetry. To verify this it suffices to consider intersections of $\left(-K_{X}\right)$ with the edges of the Mori cone. One of the edges corresponds to the Del Pezzo fibration and intersects $\left(-K_{X}\right)$ positively and the other edge corresponds to the flop and has zero intersection.

Let $D$ be a fiber of $\alpha$. The divisors $\left(-K_{X}\right)$ and $D$ form a basis in the vector space $\operatorname{Pic} X \otimes \mathbb{R}$. The strict transform of $K_{X}$ through $\phi$ is $K_{X^{\prime}}$. Since $\phi$ is a flop, $\left(-K_{X}\right)^{3}=\left(-K_{X^{\prime}}\right)^{3}$. Denote by $D^{\prime}$ the strict transform of $D$ and by $L^{\prime}$ a fiber of $\alpha^{\prime}$. The divisors $\left(-K_{X^{\prime}}\right)$ and $D^{\prime}$ form a basis for Pic $X^{\prime} \otimes \mathbb{R}$. Then $L^{\prime} \equiv y\left(-K_{X^{\prime}}\right)-x D^{\prime}$ for some real $x$ and $y$. 
Theorem 1.3. The anti-canonical divisor $\left(-K_{X}\right)$ is big.

Proof. The divisor $\left(-K_{X}\right)$ is nef and it is sufficient to prove that $\left(-K_{X}\right)^{3}>0$ or $\left(-K_{X}\right)^{3} \neq 0$.

Consider $\left(D^{\prime}\right)^{2}\left(-K_{X^{\prime}}\right)$. Choose two divisors $D_{1}, D_{2} \in|D|$. We have $D_{1} \cap$ $D_{2}=\emptyset$. Then their strict transforms $D_{1}^{\prime}$ and $D_{2}^{\prime}$ intersect in an effective 1-cycle with support in the set of indeterminacy of $\phi^{-1}$. Since we have a flop, every curve of indeterminacy has zero intersection with the canonical class $K_{X^{\prime}}$ and $\left(D^{\prime}\right)^{2}\left(-K_{X^{\prime}}\right)=0$.

Note that one has $x>0$ in the equation $L^{\prime} \equiv y\left(-K_{X^{\prime}}\right)-x D^{\prime}$. Indeed, let $R$ and $R^{\prime}$ be the edges of Mori cones corresponding to flops for $X$ and $X^{\prime}$ respectively. By considering intersection with the canonical divisor we see that flopping curves of $X$ cannot lie in fibers of $\alpha$. Thus $D \cdot R>0$ and $D^{\prime} \cdot R^{\prime}<0$. Similarly, $L^{\prime} \cdot R^{\prime}>0$ and since $K_{X^{\prime}} \cdot R^{\prime}=0$ we have $x>0$.

Now assume $\left(-K_{X}\right)^{3}=0$. The degree of a general fiber of $\alpha^{\prime}$ is $d^{\prime}=\operatorname{deg} L^{\prime}{ }_{w}=$ $K_{L^{\prime}}^{2}=L^{\prime} K_{X^{\prime}}^{2}$. It is well known that $1 \leq d^{\prime} \leq 9, d^{\prime} \neq 7$. Then $d^{\prime}=L^{\prime} K_{X^{\prime}}^{2}=$ $-x D^{\prime} K_{X^{\prime}}^{2}-y K_{X^{\prime}}^{3}=-x D^{\prime} K_{X^{\prime}}^{2} \neq 0$. At the same time $L^{\prime 2} \equiv 0$ and $L^{\prime 2} K_{X^{\prime}}=0$, that is $x^{2}\left(D^{\prime}\right)^{2} K_{X^{\prime}}+2 x y D^{\prime} K_{X^{\prime}}^{2}+y^{2} K_{X^{\prime}}^{3}=2 x y D^{\prime} K_{X^{\prime}}^{2}=0$. Therefore, $y=0$ and $L^{\prime} \equiv-x D^{\prime}$ or $L^{\prime}+x D^{\prime} \equiv 0$. Since $x>0$, the divisor from the left-hand side of the last equation is effective, which is impossible because intersection with the ample divisor $H$ gives $\left(L^{\prime}+x D^{\prime}\right) H^{2}=0$. This contradiction proves the theorem.

Corollary 1.4. (i) $h^{i}\left(\mathcal{O}_{X}\left(-m K_{X}\right)\right)=0$ for every $m \in \mathbb{Z}, i=1,2$; moreover $h^{i}\left(\mathcal{O}_{X}\left(-m K_{X}\right)\right)=0$ if $i>0, m \geq 0$ and if $i<3, m<0$. In particular, $h^{i}\left(\mathcal{O}_{X}\right)=0$ for $i>0$.

(ii) $h^{0}\left(\mathcal{O}_{X}\left(-m K_{X}\right)\right)=\frac{m(m+1)(2 m+1)}{12}\left(-K_{X}^{3}\right)+2 m+1$, in particular,

$$
h^{0}\left(\mathcal{O}_{X}\left(-K_{X}\right)\right)=\frac{\left(-K_{X}\right)^{3}}{2}+3 \geq 4
$$

and $\left(-K_{X}\right)^{3}$ is even.

Corollary 1.5. In the notation of the Theorem, $L^{\prime} \equiv y\left(-K_{X^{\prime}}\right)-x D^{\prime}$, where $x>0$, $y>0$. In addition,

$$
\begin{array}{cc}
x, y \in \mathbb{N}, & \text { if } d=1,2, \ldots, 6 ; \\
x, y \in \frac{1}{2} \mathbb{N}, & \text { if } d=8 ; \\
x, y \in \frac{1}{3} \mathbb{N}, & \text { if } d=9 .
\end{array}
$$

Proof. We will prove only $y>0$. The fact that $x>0$ was shown in the proof of the Theorem; the case $y=0$ was also considered there. As $L^{\prime 2} K_{X^{\prime}}=0$ we have $y^{2}\left(-K_{X^{\prime}}\right)^{3}-2 y x\left(-K_{X^{\prime}}\right)^{2} D^{\prime}+x^{2}\left(-K_{X^{\prime}}\right)\left(D^{\prime}\right)^{2}=0$. The first summand is positive, the last summand is zero. $\left(-K_{X^{\prime}}\right)^{2} D^{\prime}=d^{\prime}>0$ and $x>0$, therefore $y>0$.

\section{NumericAl CONSTRAints}

Denote $k=\left(-K_{X}\right)^{3}, d=\operatorname{deg} D_{w}$ the degree of the Del Pezzo fibration on $X$ and $d^{\prime}=\operatorname{deg} L_{w}^{\prime}$ the degree of the Del Pezzo fibration on $X^{\prime}$. There are two systems of 
equations:

$$
\left\{\begin{array} { l } 
{ D ^ { 3 } = 0 , } \\
{ D ^ { 2 } K _ { X } = 0 , } \\
{ D K _ { X } ^ { 2 } = d , } \\
{ K _ { X } ^ { 3 } = - k . }
\end{array} \quad \left\{\begin{array}{l}
L^{\prime 3}=0, \\
L^{\prime 2} K_{X^{\prime}}=0, \\
L^{\prime} K_{X^{\prime}}^{2}=d^{\prime} \\
K_{X^{\prime}}^{3}=-k
\end{array}\right.\right.
$$

We will find constraints on the coefficients $x$ and $y$ in the relation $L^{\prime} \equiv y\left(-K_{X^{\prime}}\right)-$ $x D^{\prime}$. We already know that $\left(D^{\prime}\right)^{2} K_{X^{\prime}}=0$. Let us denote $\left(-D^{\prime}\right)^{3}=m$ and find $D^{\prime} K_{X^{\prime}}^{2}$. Consider $\bar{X}$ with morphisms $\pi: \bar{X} \rightarrow X$ and $\pi^{\prime}: \bar{X} \rightarrow X^{\prime}$ resolving the indeterminacy of $\phi$. By the projection formula $D K_{X}^{2}=\left(\pi^{*} D\right)\left(\pi^{*} K_{X}\right)^{2}, D^{\prime} K_{X^{\prime}}^{2}=$ $\left(\pi^{\prime *} D^{\prime}\right)\left(\pi^{\prime *} K_{X^{\prime}}\right)^{2}$. Since $\phi$ is a flop, $\pi^{*} K_{X}=\pi^{\prime *} K_{X^{\prime}}$. Let $E_{1}, E_{2}, \ldots, E_{n}$ be the exceptional divisors for $\pi$; at the same time they are exceptional divisors for $\pi^{\prime}$. Let $\bar{D}$ be the strict transform for both $D$ and $D^{\prime}$. Then $\pi^{*} D=\bar{D}+\sum_{i=1}^{n} a_{i} E_{i}$ and $\pi^{\prime *} D^{\prime}=\bar{D}+\sum_{i=1}^{n} b_{i} E_{i}$. Then the difference

$$
D^{\prime} K_{X^{\prime}}^{2}-D K_{X}^{2}=\left(\pi^{*} K_{X}\right)^{2}\left(\pi^{\prime *} D^{\prime}-\pi^{*} D\right)=\left(\pi^{*} K_{X}\right)^{2}\left(\sum_{i=1}^{n}\left(b_{i}-a_{i}\right) E_{i}\right)=0 .
$$

Therefore, $D^{\prime} K_{X^{\prime}}^{2}=d$.

Now we can rewrite the second system of equations as

$$
\left\{\begin{array}{l}
y^{3} k-3 x y^{2} d+x^{3} m=0, \\
y^{2} k-2 x y d=0, \\
y k-x d=d^{\prime}, \\
k=k .
\end{array}\right.
$$

With a new parameter $t=\frac{x}{y}$ the first two equations give

$$
\left\{\begin{array}{l}
t^{3} m-3 t d+k=0, \\
2 t d-k=0
\end{array}\right.
$$

Also, after eliminating $t$ we obtain the relation $\left(\frac{k}{2}\right)^{2} m=d^{3}$. Thus, $m>0$ and this is an equation in natural numbers with $1 \leq d \leq 9, d \neq 7$. The following table contains all the solutions:

\begin{tabular}{||r|r|r||r|r|r||r|r|r||}
\hline$\frac{k}{2}$ & $m$ & $d$ & $\frac{k}{2}$ & $m$ & $d$ & $\frac{k}{2}$ & $m$ & $d$ \\
\hline 27 & 1 & 9 & 4 & 32 & 8 & 1 & 1 & 1 \\
16 & 2 & 8 & 3 & 3 & 3 & 1 & 8 & 2 \\
9 & 9 & 9 & 3 & 24 & 6 & 1 & 27 & 3 \\
8 & 1 & 4 & 3 & 81 & 9 & 1 & 64 & 4 \\
8 & 8 & 8 & 2 & 2 & 2 & 1 & 125 & 5 \\
6 & 6 & 6 & 2 & 16 & 4 & 1 & 216 & 6 \\
5 & 5 & 5 & 2 & 54 & 6 & 1 & 512 & 8 \\
4 & 4 & 4 & 2 & 128 & 8 & 1 & 729 & 9 \\
\hline
\end{tabular}

Lemma 2.1. The Riemann-Roch formula gives

$$
\chi\left(\mathcal{O}_{X}\left(a\left(-K_{X}\right)+b D\right)\right)=k \frac{2 a^{3}+3 a^{2}+a}{12}+d \frac{\left(a^{2}+a\right) b}{2}+(2 a+b)+1 .
$$


Let $L \in$ Pic $X$ be the strict transform of $L^{\prime}$. Below, the most important linear system on $X$ will be $|L|$.

Proposition 2.2. $h^{i}\left(X, \mathcal{O}_{X}(L)\right)=0$ for $i>0$.

Proof. Consider the exact sequence of sheaves

$$
0 \longrightarrow \mathcal{O}_{X} \longrightarrow \mathcal{O}_{X}(L) \longrightarrow \mathcal{O}_{L}(L) \longrightarrow 0 .
$$

As $\left(-K_{X}\right)$ is big, one has

$$
H^{1}\left(X, \mathcal{O}_{X}\right)=H^{2}\left(X, \mathcal{O}_{X}\right)=H^{3}\left(X, \mathcal{O}_{X}\right)=0, \quad H^{3}\left(X, \mathcal{O}_{X}(L)\right)=0
$$

and

$$
H^{i}\left(X, \mathcal{O}_{X}(L)\right) \simeq H^{i}\left(L, \mathcal{O}_{L}(L)\right) \text { for } i=1,2 .
$$

$L$ is a divisor on $X$, thus locally it is a complete intersection. By [Ha], Proposition 8.23 , Ch. II, $L$ is a Cohen-Macaulay scheme, and $\omega_{L}$ is a dualizing sheaf Ha, Cor. 7.7, 7.12, Ch. III]. Then for $L$ we have Serre duality

$$
H^{2}\left(L, \mathcal{O}_{L}(L)\right)^{*} \simeq H^{0}\left(L, \mathcal{O}_{L}(-L) \otimes \omega_{L}\right) \simeq H^{0}\left(L, \mathcal{O}_{L}\left(\left.K_{X}\right|_{L}\right)\right) .
$$

However, $K_{X}$ has nonnegative intersection only with the flopping curves. Then

$$
H^{2}\left(X, \mathcal{O}_{X}(L)\right)^{*} \simeq H^{0}\left(L, \mathcal{O}_{L}\left(\left.K_{X}\right|_{L}\right)\right)=0 .
$$

Note that $h^{0}\left(L, \mathcal{O}_{L}(L)\right)=1$ because the intersection of any two elements of $|L|$ lies in the flopping curves and is fixed on an arbitrary element $L$. From RiemannRoch one obtains

$$
\begin{aligned}
\chi\left(\mathcal{O}_{L}(L)\right) & =1-h^{1}\left(L, \mathcal{O}_{L}(L)\right)+0 \\
& =\chi\left(\mathcal{O}_{L}\right)+\frac{\mathcal{O}_{L}(L) \cdot \mathcal{O}_{L}(L)-\mathcal{O}_{L}(L) \cdot \omega_{L}^{\circ}}{2}=\chi\left(\mathcal{O}_{L}\right)+\left.\frac{1}{2} L \cdot K_{X}\right|_{L} .
\end{aligned}
$$

But $L$ is a rational surface, so $\chi\left(\mathcal{O}_{L}\right)=1$, and

$$
\left.L K_{X}\right|_{L}=L^{2} K_{X}=L^{\prime 2} K_{X^{\prime}}=0 .
$$

Therefore, $h^{1}\left(L, \mathcal{O}_{L}(L)\right)=0$ and $h^{1}\left(X, \mathcal{O}_{X}(L)\right)=0$.

Corollary 2.3. $h^{0}\left(X, \mathcal{O}_{X}(L)\right)=k \frac{2 y^{3}+3 y^{2}+y}{12}-d \frac{\left(y^{2}+y\right) x}{2}+(2 y-x)+1$.

Theorem 2.4. The degrees of the two Del Pezzo fibrations coincide.

Proof. As earlier $L^{\prime} \equiv y\left(-K_{X^{\prime}}\right)-x D^{\prime}, L \equiv y\left(-K_{X}\right)-x D$ and let $D \equiv y^{\prime}\left(-K_{X}\right)-$ $x^{\prime} L$. Then $D \equiv y^{\prime}\left(-K_{X}\right)-x^{\prime}\left(y\left(-K_{X}\right)-x D\right)$ and

$$
\left\{\begin{array}{l}
x^{\prime} x=1, \\
y^{\prime}-x^{\prime} y=0 .
\end{array}\right.
$$

Here $x=\frac{1}{3}, \frac{1}{2}, \frac{2}{3}, 1, \frac{3}{2}, 2,3$ and $x^{\prime}=3,2, \frac{3}{2}, 1, \frac{2}{3}, \frac{1}{2}, \frac{1}{3}$ by Corollary 1.5, But $d^{\prime}=$ $y k-x d=(y k-2 x d)+x d=x d$ and it is sufficient to prove that $x=x^{\prime}=1$. By interchanging $X$ and $X^{\prime}$ it is enough to reject only the cases $x=\frac{1}{3}, \frac{1}{2}, \frac{2}{3}$ for which $d=9,8,9$, respectively. If $x=\frac{2}{3}$, then $d=9, d^{\prime}=6, x^{\prime}$ is natural and $x^{\prime} \neq \frac{3}{2}$. If $x=\frac{1}{3}$ or $\frac{1}{2}$, then $y=\frac{2 x d}{k}$ and by Corollary 2.3 we can check that the linear system $|L|$ is not a pencil. Using the table above this assertion is easy to prove by exhaustion. 
Now we can complete the proof of the main classification Theorem 0.1 . Use Corollary 2.3 again. Now $x=1$ and $d=\left(\frac{k}{2}\right) y$. We have

$$
2=h^{0}\left(X, \mathcal{O}_{X}(L)\right)=\left(\frac{k}{2}\right) \frac{(2 y+1)(y+1) y}{6}-\left(\frac{k}{2}\right) \frac{(y+1) y^{2}}{2}+2 y
$$

or

$$
\left(\frac{k}{2}\right) \frac{(y+1) y(y-1)}{6}=2 y-2 .
$$

This means that $y=1$ or $\frac{k}{2}=\frac{12}{(y+1) y}$. As $\frac{k}{2} \in \mathbb{N}$ and $y=\frac{2 d}{k}$ we obtain a new shorter list of possibilities.

\begin{tabular}{|r|r|r|r|}
\hline$\frac{k}{2}$ & $m$ & $d$ & Linear system $|L|$ \\
\hline 27 & 1 & 9 & $\left|\frac{1}{3}\left(-K_{X}\right)-D\right|$ \\
16 & 2 & 8 & $\left|\frac{1}{2}\left(-K_{X}\right)-D\right|$ \\
$n$ & $n$ & $n$ & $\left|\left(-K_{X}\right)-D\right| \quad$ for $1 \leq n \leq 9, n \neq 7$. \\
2 & 16 & 4 & $\left|2\left(-K_{X}\right)-D\right|$ \\
1 & 27 & 3 & $\left|3\left(-K_{X}\right)-D\right|$ \\
\hline
\end{tabular}

This proves Theorem 0.1

\section{EXAmples}

The purpose of this section is to construct examples of links for 9 of the 12 numerical possibilities of Theorem 0.1 .

Example 3.1. $K_{X}^{3}=-54, d=9$. Consider the variety

$$
\bar{X}_{1}=\mathbb{P}_{\mathbb{P}^{1} \times \mathbb{P}^{1}}(\mathcal{O} \oplus \mathcal{O}(-1,-1)) .
$$

We have the exceptional section $E$ of the $\mathbb{P}^{1}$-fibration. $E \simeq \mathbb{P}^{1} \times \mathbb{P}^{1}$ and the divisor $E$ is contractible along each of its generators. After such contractions we will get two smooth varieties $X_{1}$ and $X_{1}^{\prime}$. We have a flop in the image of $E$ which is a rational curve $Z \simeq \mathbb{P}^{1}$ with the normal bundle $\mathcal{N}_{Z / X_{1}} \simeq \mathcal{O}_{\mathbb{P}^{1}}(-1) \oplus \mathcal{O}_{\mathbb{P}^{1}}(-1)$. Also, we have two Del Pezzo fibrations $\alpha_{1}: X_{1} \rightarrow \mathbb{P}^{1}$ and $\alpha_{1}^{\prime}: X_{1}^{\prime} \rightarrow \mathbb{P}^{1}$ with all fibers isomorphic to $\mathbb{P}^{2}$.

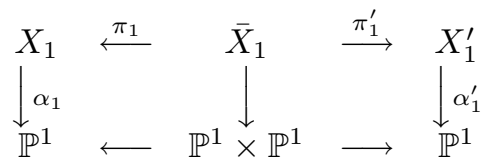

Example 3.2. $K_{X}^{3}=-32, d=8$. Choose a section $S$ of the $\mathbb{P}^{1}$-bundle $\bar{X}_{1}$ from Example 3.1 such that $S \cap E=\emptyset$. Choose a smooth curve $C$ of bidegree $(2,2)$ on $S \simeq \mathbb{P}^{1} \times \mathbb{P}^{1}$. Consider $\bar{X}_{2}$ which is the blowing up of $\bar{X}_{1}$ along $C$. Let $S_{2}$ and $E_{2}$ be the strict transforms of $S$ and $E$. Then $S_{2}, E_{2} \simeq \mathbb{P}^{1} \times \mathbb{P}^{1}$ and they are contractible along each of the generators. There are four different ways to contract both divisors but only two lead to algebraic varieties. Denote this pair of varieties by $X_{2}$ and $X_{2}^{\prime}$. We have a flop in two isolated rational curves with the normal bundles isomorphic to $\mathcal{O}_{\mathbb{P}^{1}}(-1) \oplus \mathcal{O}_{\mathbb{P}^{1}}(-1)$. Both Del Pezzo fibrations have quadrics as general fiber. 
Example 3.3. $K_{X}^{3}=-2, d=3$. Consider $P=\mathbb{P}^{1} \times \mathbb{P}^{3}$ with projections $p_{1}: P \rightarrow$ $\mathbb{P}^{1}, p_{2}: P \rightarrow \mathbb{P}^{3}$ and coordinates $\left(x_{0}: x_{1}, y_{0}: y_{1}: y_{2}: y_{3}\right)$. If $A$ is a point in $\mathbb{P}^{1}$ and $H$ is a plane in $\mathbb{P}^{3}$, then denote $F=p_{1}^{*} A$ and $M=p_{2}^{*} H$. Let the divisor $X_{3} \sim 3 M+2 F$ be given by an equation $x_{0}^{2} f(y)+x_{0} x_{1} g(y)+x_{1}^{2} h(y)=0$, where $f, g, h$ are homogeneous cubic forms of $y_{0}, y_{1}, y_{2}, y_{3}$, such that the three corresponding cubics in $\mathbb{P}^{3}$ intersect only in 27 distinct points $Q_{1}, Q_{2}, \ldots, Q_{27}$ and the variety $X_{3}$ is smooth. General cubics satisfy these conditions and one example is

$$
\left\{\begin{array}{l}
f=y_{0}^{3}+y_{1}^{3}-y_{2}^{3}-y_{3}^{3}, \\
g=y_{0}^{3}-y_{1}^{3}+y_{2}^{3}-y_{3}^{3}, \\
h=y_{0}^{3}-y_{1}^{3}-y_{2}^{3}+y_{3}^{3} .
\end{array}\right.
$$

Then $X_{3}$ has a natural Del Pezzo fibration of degree 3 which is given by the restriction $\left.p_{1}\right|_{X_{3}}: X_{3} \rightarrow \mathbb{P}^{1}$. As usual, we denote the class of a fiber by $D$.

$$
K_{X_{3}}=\left.\left(K_{P}+X_{3}\right)\right|_{X_{3}}=\left.((-4 M-2 F)+(3 M+2 F))\right|_{X_{3}}=-\left.M\right|_{X_{3}} .
$$

Then $-K_{X_{3}}$ is nef and it is big because $\left(-K_{X_{3}}\right)^{3}=M^{3}(3 M+2 F)=2$. The only curves in $X_{3}$ that can have non-positive intersection with the anti-canonical class are those mapped by $p_{2}$ to a point. If $y=\left(y_{0}: y_{1}: y_{2}: y_{3}\right)$ are coordinates of such a point, then $x_{0}^{2} f(y)+x_{0} x_{1} g(y)+x_{1}^{2} h(y)=0$ for all $\left(x_{0}: x_{1}\right)$. This implies $f(y)=g(y)=h(y)=0$. There are 27 such curves $C_{1}, C_{2}, \ldots, C_{27}$ over the points $Q_{1}, Q_{2}, \ldots, Q_{27}$.

Consider divisors $B_{a}$ on $X_{3}$ which are determined by the equations $a_{0}^{2} f(y)$ $+a_{0} a_{1} g(y)+a_{1}^{2} h(y)=0$ with the parameter $\left(a_{0}: a_{1}\right)$. Each of them contains the fiber $D_{a}$ over the point $\left(a_{0}: a_{1}\right) \in \mathbb{P}^{1}$ as one of irreducible components. Denote by $L_{a}$ the other part of $B_{a}$. Then $\left.L_{a} \sim(3 M-F)\right|_{X_{3}}$ and $L_{a}^{2}\left(-K_{X_{3}}\right)=$ $(3 M-F)^{2} M(3 M+2 F)=0$. Therefore, for any $L_{1}, L_{2} \in\left|L_{a}\right|$ the intersection $L_{1} \cap L_{2}$ consists of precisely the curves $C_{i}$ with constant multiplicities. Considering $L_{1}$ for $a=(1: 0)$ and $L_{2}$ for $a=(0: 1)$ shows that all multiplicities are 1 .

The curves $C_{1}, C_{2}, \ldots, C_{27}$ contract by $p_{2}$ to the points $Q_{1}, Q_{2}, \ldots, Q_{27}$. Over $\mathbb{P}^{3} \backslash \bigcup_{i=1}^{27} Q_{i}$ the projections $p_{2}: B_{a} \rightarrow p_{2}\left(B_{a}\right)$ are $2-1$, so the projections $p_{2}:$ $L_{a} \rightarrow p_{2}\left(L_{a}\right)$ are 1-1. The image $p_{2}\left(L_{a}\right)$ is a smooth cubic for general $a$. The $C_{i}$ are (-1)-curves on $L_{a}$ and the only possibility for the normal bundles $\mathcal{N}_{C_{i} / X_{3}}$ is $\mathcal{N}_{C_{i} / X_{3}} \simeq \mathcal{O}_{\mathbb{P}^{1}}(-1) \oplus \mathcal{O}_{\mathbb{P}^{1}}(-1)$. After the flop in the curves $C_{1}, C_{2}, \ldots, C_{27}$ the transforms of $L_{a}$ become fibers of a Del Pezzo fibration of degree 3.

All the following examples will be similar to Example 3.3 Even Examples 3.1 and 3.2 can be described in the same way.

Example 3.4. $K_{X}^{3}=-6, d=3$. Let $C$ be a smooth cubic in $P=\mathbb{P}^{4}$. Consider $P=\mathbb{P}^{1} \times C$ with projections $p_{1}: P \rightarrow \mathbb{P}^{1}, p_{2}: P \rightarrow C$. Let $A$ be a point in $\mathbb{P}^{1}$ and $H$ be an ample generator of $\operatorname{Pic} C \simeq \mathbb{Z} H$. $H$ is an intersection of $C$ and a hyperplane in the ambient space $P=\mathbb{P}^{4}$. As in the previous example we denote $F=p_{1}^{*} A$ and $M=p_{2}^{*} H$. Take a general smooth divisor $X_{4} \sim M+2 F . X_{4}$ has the structure of a degree 3 Del Pezzo fibration. We have

$$
K_{X_{4}}=\left.\left(K_{P}+X_{4}\right)\right|_{X_{4}}=\left.((-2 M-2 F)+(M+2 F))\right|_{X_{4}}=-\left.M\right|_{X_{4}} .
$$

Therefore, $-K_{X_{4}}$ is nef and big with $\left(-K_{X_{4}}\right)^{3}=M^{3}(2 M+2 F)=2 \times 3=6$. A curve in $X_{4}$ can have non-positive intersection with the anti-canonical class if and only if the curve is mapped by $p_{2}$ to a point. There are 3 such curves $C_{1}, C_{2}, C_{3}$. We will have a flop in these curves. 
For every fiber $D_{a}$ over a point $\left(a_{0}: a_{1}\right) \in \mathbb{P}^{1}$ define the divisor $B_{a}=p_{2}^{*}\left(p_{2 *} D_{a}\right)$ on $X_{3} . B_{a}$ contains $D_{a}$ as one of its irreducible components. Denote by $L_{a}$ the other part of $B_{a}$. Then $\left.L_{a} \sim(M-F)\right|_{X_{3}}$ and

$$
L_{a}^{2}\left(-K_{X_{3}}\right)=(M-F)^{2} M(M+2 F)=0 .
$$

Similar to the previous Example we get a flop in curves $C_{1}, C_{2}, C_{3}$ and after the flop transforms of $L_{a}$ become fibers of a Del Pezzo fibration of degree 3.

Example 3.5. $K_{X}^{3}=-4, d=2$. Example 3.4 is based on the fact that the cubic $C=V_{3}$ is a Fano variety of index 2 , that is $-K_{C}=2 H$ where Pic $C \simeq \mathbb{Z} H$. For this Example use instead of the cubic $C$ a Fano variety $V_{2}$ of index 2 with $K_{V_{2}}{ }^{3}=-16$. We can choose $X_{5} \sim M+2 F$ to be smooth because the linear system $|M+2 F|$ is base point free.

Example 3.6. $K_{X}^{3}=-8, d=4$. Replace the cubic $C$ in Example 3.4 by an index 2 Fano variety $V_{4}$ with $K_{V_{4}}{ }^{3}=-32$.

Example 3.7. $K_{X}^{3}=-10, d=5$. Replace the cubic $C$ in Example 3.4 by an index 2 Fano variety $V_{5}$ with $K_{V_{5}}{ }^{3}=-40$.

Example 3.8. $K_{X}^{3}=-4, d=4$. Replace the cubic $C$ in Example 3.4 by a quadric $Q \in \mathbb{P}^{4}$. Let $H \sim\left(-\frac{1}{3} K_{Q}\right)$, and $M$ and $F$ be defined as in Example 3.4. Take $X_{8} \sim 2 M+2 F$.

Example 3.9. $K_{X}^{3}=-16, d=8$. Replace the cubic $C$ in Example 3.4 by a projective space $\mathbb{P}^{3}$. Let $H$ be a hyperplane on $\mathbb{P}^{3}$, and $M$ and $F$ be defined as in Example 3.4 Take $X_{9} \sim 2 M+2 F$.

\section{ACKNOWLEDGMENTS}

The author would like to thank Professors V. Iskovskikh, V. Alexeev and R. Varley.

\section{REFERENCES}

[Cor] A.Corti, Factoring birational maps of threefold after Sarkisov, J. Alg. Geometry, 4 (1993), 223-254. MR 96c:14013

[Ha] R.Hartshorne, Algebraic Geometry, Springer-Verlag, NewYork-Heidelberg-Berlin, 1977. MR 57:3116

[MM] S.Mori, S.Mukai, Classification of Fano threefolds with $B_{2} \geq 2$, Manuscripta Math., 36 (1981), 147-162. MR 83f:14032

[Re] M.Reid, Birational geometry of 3-folds according to Sarkisov, preprint (1991).

[Sar] V.G.Sarkisov, Birational maps of standard $\mathbb{Q}$-Fano fiberings, I.V.Kurchatov Institute Atomic Energy preprint (1989).

Department of Mathematics, University of Georgia, Athens, Georgia 30602

E-mail address: vologods@math.uga.edu 